breast cancer risk factors which are hardly modifiable, the identification of occupational or environmental risk factors may be a key to prevention. It has been suggested that exposure to organic solvents in the workplace may play a role in the etiology of breast cancer. However, most epidemiological studies on solvents in female breast cancer have reported inconsistent results.

Methods We examined the risk of breast cancer related to lifetime exposure to chlorinated, petroleum-based and oxygenated solvents in the CECILE study, a large population-based casecontrol study carried out in France (2005-2007). 1230 women with breast cancer and 1315 population controls were included. Data collected included sociodemographic characteristics, medical and family history, anthropometric measurements, reproductive history, lifestyle habits, and lifetime occupational history. All jobs were coded using the International Standard Classification of Occupations (ISCO 1968) and the Classification of Activities in the European Community (NACE 1991). Exposure to five chlorinated, five petroleum and five oxygenated solvents was assessed using job-exposure matrices, which assigned indices of exposure (probability, frequency and intensity) for each job. A Cumulative Exposure Score (CES) taking into account probability, frequency, intensity and duration of exposure was calculated for each subject. Odds ratio (ORs) and their 95\% confidence intervals (95\% $\mathrm{CI})$ were estimated using unconditional logistic regression adjusting for potential confounders. Analyses were stratified by the menopausal status.

Results Significantly increased ORs were found in premenopausal women with high probability of exposure to alcohols $(1.50 ; 1.05-2.14)$ and diethyl ether $(1.97 ; 1.00-3.88)$. Premenopausal women with the highest CES of oxygenated solvents had an $\mathrm{OR}$ of $1.70(1.09-2.64)(\mathrm{OR}=1.67 ; 1.10-2.52$ for alcohols, 1.80 ; $0.95-3.42$ for diethyl ether); the highest CES of chlorinated solvents was associated with an OR of 2.14 (0.86-5.30). In premenopausal women, a duration of exposure to oxygenated solvents $\geq 25$ years with a probability of exposure $\geq 80 \%$ significantly increased the risk of breast cancer $(\mathrm{OR}=2.62 ; 1.27-5.40$ for all solvents, $2.72 ; 1.30-5.70$ for alcohols, and 2.79; 0.67-11.6 for diethyl ether). No association was observed for oxygenated, petroleum-based or chlorinated solvents in postmenopausal women.

Conclusion These findings suggest a role of occupational exposure to oxygenated solvents, notably to alcohols and diethyl ether, in the occurrence of breast cancer in premenopausal women. Our results highlight the importance of timing and duration of exposure for the study of occupational exposure to solvents in breast cancer risk.

\section{RF05 THE IMPACT OF DISTRIBUTION SHIFTS IN A POPULATION'S CARDIOVASCULAR RISK FACTORS ON HEALTHY LIFE EXPECTANCY IN JAPAN}

\begin{abstract}
${ }^{1} \mathrm{R}$ Tsukinoki ${ }^{*},{ }^{2} \mathrm{Y}$ Murakami, ${ }^{3,4} \mathrm{~K}$ Miura, ${ }^{5} \mathrm{~T}$ Okamura, ${ }^{3,4} \mathrm{~A}$ Kadota, ${ }^{6} \mathrm{~T}$ Hayakawa, ${ }^{7} \mathrm{~A}$ Okayama, ${ }^{3,4} \mathrm{H}$ Ueshima. 'Department of Community Health Nursing, apanese Red Cross College of Nursing, Tokyo, Japan; ' Department of Medical Statistics, School of Medicine, Toho University, Tokyo, Japan; ${ }^{3}$ Department of Public Health, Shiga University of Medical Sciences, Otsu, Japan; ${ }^{4}$ Center for Epidemiologic Research in Asia, Shiga University of Medical Sciences, Otsu, Japan; ${ }^{5}$ Department of Preventive Medicine and Public Health, Keio University, Tokyo, Japan; ${ }^{6}$ The Kinugasa Research Organization, Ritsumeikan University, Kyoto, Japan; ${ }^{7}$ Research Institute of Strategy for Prevention, Tokyo, Japan
\end{abstract}

10.1136/jech-2019-SSMabstracts. 120
Background Healthy life expectancy (HLE) is an important indicator of population health. Although the distribution of risk factors within populations has shifted during the past few decades (e.g., smoking rates have declined in developed countries), little is known if these changes affect population HLE. Our study aimed to estimate the impact of distribution shifts in a population's cardiovascular risk factors on HLE in Japan. Methods Data for analysis were obtained from NIPPON DATA90, a nationwide cohort study of over 9,000 Japanese people initiated in 1990. Using activities of daily living scores, we estimated the HLEs of study participants aged $\geq 60$ years at the baseline survey. We examined shifts in the following three risk factors: systolic blood pressure (SBP), body mass index (BMI), and smoking status. All HLE calculations were performed using maximum likelihood approach with interpolated Markov chains (iMaCh 0.98r7). First, risk factor-specific HLEs were calculated using multistate life tables. A total of 24 combinations of cardiovascular risk factors were estimated. Next, we calculated the population's baseline HLE (Scenario $0)$ using the weighted average of the risk factor-specific HLEs. The averaging weight was derived from the multivariable distribution of baseline data from NIPPON DATA90. Finally, scenarios were set to investigate the impact of distribution shifts in risk factors. In Scenario 1, the distributions of SBP and BMI were reduced by $4.0 \mathrm{mmHg}$ and $2.0 \mathrm{~kg} / \mathrm{m}^{2}$, respectively. In Scenario 2, we added the condition to Scenario 1 that all current smokers who wanted to quit smoking (men: 25\%, women: 40\%) were successful in quitting.

Results The analysis was performed using 6,676 participants (2,840 men and 3,836 women). The baseline HLE at age 60 years (Scenario 0) was 20.02 years in men and 24.32 years in women. After the shift in risk factors, the adjusted HLE at age 60 years in Scenario 1 was 20.12 years in men and 24.45 years in women, and the corresponding HLE in Scenario 2 was 20.46 years in men and 24.53 years in women. When compared with the baseline HLE, Scenario 1 presented a gain of 0.10 years in men and 0.14 years in women, and Scenario 2 presented a gain of 0.43 years in men and 0.21 years in women.

Conclusion We examined the impact of distribution shifts in SBP, BMI, and smoking status on population HLE in Japan, and found that smoking cessation substantially extended HLE.

\section{RF06 CHILDREN PRESENTING WITH CHRONIC PAIN TO THE ENGLISH NATIONAL HEALTH SERVICE: A WHOLE- POPULATION ADMINISTRATIVE DATA COHORT STUDY}

${ }^{1,2}$ MA Jay* ${ }^{2}$ RF Howard, ${ }^{1}$ R Gilbert. 'Population, Policy and Practice Programme, UCL GOS Institute of Child Health, London, UK; ${ }^{2}$ Department of Anaesthesia and Pain Medicine, Great Ormond Street Hospital for Children NHS Foundation Trust, London, UK

10.1136/jech-2019-SSMabstracts. 121

Background Management of paediatric chronic pain (CP: pain $>3$ months' duration) is challenging and associated with significant comorbidities. Although large numbers of children may be affected, this population in England has not been well-described. In addition, despite partial provision of specialist NHS CP services, referral pathways are not well-established; children may therefore be receiving suboptimal interventions and access to $\mathrm{CP}$ services may be inequitable. We examined the characteristics and service utilisation of all children attending specialised CP services in England to inform commissioners and service providers. 
Methods We used the Hospital Episode Statistics - data covering all NHS-funded inpatient admissions, outpatient appointments and A\&E attendances in English hospitals. Children aged 10-17 first seen by a CP service between 2007/8 and 2016/17 were identified using service specialty codes that identify CP services. The presence of comorbid chronic conditions (e.g., cancer, neurological disorders or mental health conditions) in the five years prior to the first attended CP appointment (CP1) were identified from inpatient data using a validated ICD-10 code list. Service utilisation was described by histograms and rates of outpatient attendances, planned and emergency admissions and A\&E attendances. Analyses were conducted in R.

Results There were 3,185 (28\%) boys and 8,330 (72\%) girls. In boys, $24 \%$ lived in the most deprived fifth of neighbourhoods in the country and $17 \%$ in the least deprived fifth; in girls, no such gradient was observed with $20 \%$ in all fifths. Comorbidity prevalence was high: $55 \%$ of boys and girls had some other chronic condition in the five years prior to CP1. There was a rise in outpatient attendances, emergency admissions and A\&E attendances in the two years prior to CP1, with rises in emergency admissions and A\&E attendances most striking. For example, the rate of emergency admissions 21-24 months prior to CP1 was 11.7 (95\% CI 10.8, 12.6) per 100 person-years; in the three months prior to CP1, it was 38.3 (95\% CI 36.7, 39.9). All abated after CP1. There was a spike of planned admissions after CP1 with a flat trend beforehand.

Conclusion Children presenting to CP services exhibit high degrees of physical and mental comorbidity. We found patterns of service utilisation indicative of unmet need prior to CP1 that warrants further investigation. Though our results may not generalise to all children with $\mathrm{CP}$, this study suggests that earlier identification and referral could reduce unnecessary A\&E visits and emergency admissions.

\section{Mental Health}

\section{RF07 RELEASE OF 13 REASONS WHY AND HOSPITAL- PRESENTING SELF-HARM IN IRELAND}

1,2P Corcoran*, 'E Griffin, ${ }^{1,2} \mathrm{CB}$ Dillon, ${ }^{1} \mathrm{~N}$ McTernan, ${ }^{2} \mathrm{AP}$ Fitzgerald, ${ }^{1,2} \mathrm{E}$ Arensman. ${ }^{1}$ National Suicide Research Foundation, National Suicide Research Foundation, Cork, Ireland; ${ }^{2}$ School of Public Health, University College Cork, Cork, Ireland

\subsection{6/jech-2019-SSMabstracts. 122}

Background Portrayals of suicidal behaviour in the media have been linked with copycat suicidal behaviour, a phenomenon referred to as the Werther Effect. In recent years, media reporting of high-profile suicides and portrayals of fictional suicides in film/television have been associated with increased deaths.

The Netflix series 13 Reasons Why was released on 31 March 2017 and received a lot of public attention, particularly for its detailed portrayal of the suicide of the female protagonist. We examined whether there was any evidence of an increase in hospital-presenting self-harm in Ireland following the release of the series.

Methods The National Self-Harm Registry Ireland records selfharm presentations to all Irish hospital emergency departments. Case ascertainment and data collection are carried out by trained data registration officers who follow standard operating procedures. We examined the temporal pattern of hospital-presenting self-harm by persons aged $\geq 10$ years, stratified by sex and whether the self-harm involved sharp objects as depicted in 13 Reasons Why or other means. Poisson regression was used to compare the incidence in the three months after the series' release compared to the rest of the calendar year 2017 and to make the same comparison for each of the preceding six years.

Results During 2011-2017, there was an annual average of 11,515 self-harm presentations to hospital from an annual average population of 3.99 million persons aged. Self-harm involving sharp objects accounted for one in four presentations $(2,937$ per year, 25.5\%) and had an age-standardised female and male incidence rate of 79 and 75 per 100,000 person-years, respectively. Three quarters of presentations involved other means (8,578 per year, $74.5 \%)$, primarily intentional drug overdose, with a female and male incidence rate of 245 and 197 per 100,000 person-years, respectively.

The incidence of female self-harm involving sharp objects was 100 per 100,000 in the three months after the release of 13 Reasons Why, 20\% higher than the incidence in the rest of 2017 (incidence rate ratio, $\mathrm{IRR}=1.20 ; 95 \%$ confidence interval $=1.08-1.33$ ). Such an increased rate was not observed for the same period in any of the previous six years (IRR range $=0.95-1.07)$. The same three-month period showed no notable change in incidence in any year for male self-harm involving sharp objects or for female or male self-harm involving other means.

Conclusion The study findings show evidence of an increase in female hospital-presenting self-harm involving sharp objects following the release of the 13 Reasons Why series.

\section{RF08 PERCEIVED SEX DISCRIMINATION, MENTAL HEALTH AND WELLBEING: A PROSPECTIVE ANALYSIS OF THE UK HOUSEHOLD LONGITUDINAL STUDY}

RA Hackett*, A Steptoe, SE Jackson. Behavioural Science and Health, University College London, London, UK

\subsection{6/jech-2019-SSMabstracts.123}

Background Perceived sex discrimination is linked with poor mental health. However, previous research is dominated by small convenience samples of US students, with a focus on sexual harassment rather than discrimination more broadly. This study aimed to examine cross-sectional and prospective associations between perceived sex discrimination and mental health and wellbeing in a community sample from the United Kingdom (UK).

Methods Data were from 2956 women aged $\geq 16$ years participating in the UK Household Longitudinal Study. Perceived discrimination was reported in 2009/10. Psychological distress, mental functioning, life satisfaction and self-rated health were assessed in 2009/10 and 2013/14. Depression was assessed in 2009/10. Linear and logistic regression analyses adjusted for age, household income, education and ethnicity.

Results Perceived sex discrimination was reported by 576 (19.5\%) participants. Cross-sectionally, perceived discrimination was associated with increased depression (Odds ratio $(\mathrm{OR}=3.16$, 95\% Confidence Interval (CI) $2.10 ; 4.79)$ and psychological distress $(B=1.26,95 \%$ CI $0.95 ; 1.56)$, and poorer mental functioning $(B=-5.39,95 \%$ CI -6.33 ; -4.46), life satisfaction $(B=-0.52,95 \%$ CI $-0.69 ;-0.36)$ and self-rated health 